J. theor. Biol. (1975) 49, 439-459

\title{
Simulation of Differential Effects on Rates in Membrane Transport $\dagger$
}

\author{
Lester I. Harrison and Halvor N. Christensen \\ Department of Biological Chemistry, University of Michigan, \\ Ann Arbor, Michigan 48104, U.S.A.
}

(Received 8 March 1974, and in revised form 28 March 1974)

Solute uptake by a mobile carrier mechanism has been simulated with an analog computer for the case where the movement of the carrier across the membrane is rate-limiting. The ratio of the rate constants for the translocation of the loaded carrier to those for the translocation of the empty carrier was varied to simulate three important conditions of transport: a ratio greater than unity, which led as previously predicted to trans stimulation; a ratio less than unity, which led as predicted to trans inhibition; and a ratio equal to unity, which eliminated any effect of solute concentration on the trans flux. In addition, transients related to the relative amount of the mediator species are recorded. When previous loading of the cells with the solute was simulated, we obtained overshoots in the uptake of labeled solute corresponding to those often seen experimentally. The inhibitory action of 2,4-dinitrofluorobenzene on a well characterized laboratory example of trans stimulation was analyzed in terms of the model. Whereas the simulation shows that a change in the ratio of the rate constants for translocation can modify the intensity of the trans phenomenon, the action of dinitrofluorobenzene proved instead to arise from decreases in the two carrier fluxes that cause the transport in question to be uphill. The distribution of the influence between these two fluxes may assist identification of the mode of energization of the process.

\section{Introduction}

Many transport systems have been shown to concentrate a substrate by a trans membrane exchange for another substrate of the same system. In a few instances the question can even be raised whether the transport system accomplishes anything but exchange (Brock \& Moo-Penn, 1962; Thomas \& Christensen, 1971). For the majority of the cases, however, the exchange

† Support is acknowledged from Grant HD01223, The Institute for Child Health and Human Development, National Institutes of Health, U.S. Public Health Service. L.I.H. was a predoctoral trainee of Training Grant GM00187.

T.B. 
phenomenon is recognized to be closely associated with net transport by the same system. Exchange between solutes proves the physical separation of the entry and the exit events, and provides evidence for the existence of a transport site sequentially but not simultaneously accessible to the substrate from the two sides of the membrane. We will continue here the conventional use of the word carrier to imply the mobility thus indicated for the mediating structure, without implications as to how large a part of the structure actually moves.

Heinz \& Walsh (1958) showed that it was the trans flux (i.e., the flux towards the perturbing mass of solute) which is stimulated in an accelerative exchange (trans stimulation), and Heinz \& Durbin (1957) provided a theoretical explanation for this behavior. They showed that under the usual transport models a translocation of the solute-carrier complex more rapid than that of the empty carrier could lead to trans stimulation.

It is sometimes overlooked that Heinz \& Durbin developed in the same paper the prediction that trans inhibition would result instead, given the reverse situation that the empty carrier is translocated more rapidly than the solute-carrier complex. The term trans inhibition implies that a perturbing mass of transport substrate decreases the flux of the same substrate or its analogs across the membrane toward the perturbing mass. We subsequently showed in this laboratory that trans inhibition could be caused to replace trans stimulation in a given transport system by chemical modification of the substrate structure (Christensen, 1972).

Oxender \& Whitmore (1966) showed that the treatment of Ehrlich ascites tumor cells with 2,4-dinitroffuorobenzene could largely eliminate the vigorous trans stimulation normally shown by $\mathrm{Na}^{+}$-independent system $L$, with only minor effects on net transport by this system. This result could have implied that the exchange phenomenon is really not an inherent part of the transport process, even though inhibition analysis has suggested that it is (Christensen \& Handlogten, 1968). To us, it has seemed more likely, however, that this reagent might produce its effect by causing a slowing of the translocation of the loaded carrier, a slowing not shared proportionately by the empty carrier; thus, the reagent may modify the flux differential presumably responsible for trans stimulation. To examine the theoretical behavior of such systems, we have tested the effects of changing each of the translocation rate constants under analog computer simulation.

The simulation results substantiate the prediction that a differential slowing of the translocation of the two carrier species can decrease or increase trans stimulation. The laboratory test of the effect of dinitrofluorobenzene on amino acid transport showed, however, that the reagent appears instead to decrease the asymmetry between the rate constants that account for the uphill character 
of the transport. Accordingly, an interference with energization appears more likely.

The results of the computer simulation are in agreement with the mathematical treatments of carrier transport provided by Stein (1967), Geck (1971), Britton (1965), and Kotyk (1973), among others, and involve no new assumptions. The simulation has the advantage of allowing us an easy manipulation of the mathematical model not only to obtain solutions under complex conditions but also to test for good fit between theoretical and experimentally determined values.

\section{The Mobile Carrier Model}

Figure 1 illustrates a conventional carrier transport mechanism which can apply equally well to active or passive transport. The model involves the binding of the solute to a carrier at the membrane surface (side 1), the move-

$s_{1}$

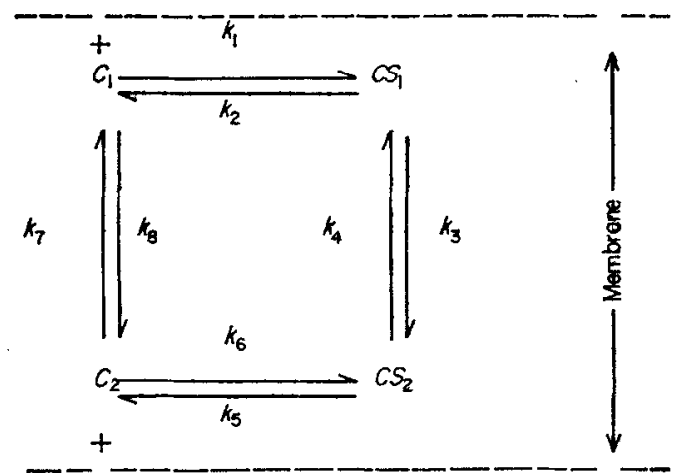

$S_{2}$

\begin{tabular}{|c|c|c|}
\hline Condition & $\begin{array}{l}\text { Asymmetry generoted } \\
\text { in distribution of } \\
\text { carrier }\end{array}$ & $\begin{array}{l}\text { Flux asymmetry } \\
\text { coused by } \\
\text { solute }\end{array}$ \\
\hline$k_{3}=k_{4}=k_{7}=k_{8}$ & (None) & None \\
\hline II. $\quad k_{3}=k_{4}>k_{7}=k_{6}$ & $C_{2}>C_{1}$ & $\begin{array}{l}\text { trans } \\
\text { stimulation }\end{array}$ \\
\hline I. $k_{7}=k_{8}>k_{3}=k_{4}$ & $(C S)_{1}>(C S)_{2}$ & $\begin{array}{l}\text { trons } \\
\text { inhibition }\end{array}$ \\
\hline
\end{tabular}

FIG. 1. Proposed model of mediated transport. $S_{\mathrm{z}}$ and $S_{\mathrm{a}}$ are the solute concentrations on the outside and on the inside of the membrane respectively, and $C$ is a mobile carrier. The model can apply either to an active transport if an energy input is provided, or to a facilitated diffusion. 
ment of the carrier-solute complex across the membrane, and the release of the solute on the other side (side 2). Distribution of the solute and the carrier species can be described in terms of the model by differential equations (1) through (5):

$$
\begin{aligned}
& \frac{\mathrm{d}[C]_{1}}{\mathrm{~d} t}=k_{2}[C S]_{1}+k_{7}[C]_{2}-\left(k_{1}[S]_{1}+k_{8}\right)[C]_{1} \\
& \frac{\mathrm{d}[C S]_{1}}{\mathrm{~d} t}=k_{1}[S]_{1}[C]_{1}+k_{4}[C S]_{2}-\left(k_{2}+k_{3}\right)[C S]_{1} \\
& \frac{\mathrm{d}[C S]_{2}}{\mathrm{~d} t}=k_{3}[C S]_{1}+k_{6}[S]_{2}[C]_{2}-\left(k_{4}+k_{5}\right)[C S]_{2} \\
& \frac{\mathrm{d}[C]_{2}}{\mathrm{~d} t}=k_{5}[C S]_{2}+k_{8}[C]_{1}-\left(k_{6}[S]_{2}+k_{7}\right)[C]_{2} \\
& \frac{\mathrm{d}[S]_{2}}{\mathrm{~d} t}=k_{5}[C S]_{2}-k_{6}[S]_{2}[C]_{2}
\end{aligned}
$$

and the mass-balance equation

$$
C_{\text {total }}=[C]_{1}+[C S]_{1}+[C S]_{2}+[C]_{2} \text {. }
$$

Solution of these equations can be simplified by the following conventional restriction: Reaction of the solute with the carrier at either membrane surface will be assumed to proceed at a much faster rate than the movement of either the loaded or the empty carrier across the membrane. In terms of the model, the magnitudes of the rate constants $k_{1}, k_{2}, k_{5}$ and $k_{6}$ are assumed much greater than those of $k_{3}, k_{4}, k_{7}$ and $k_{8}$.

We will call a passive transport in which the loaded and the unloaded carrier show the same translocation rate constant, i.e., in which $k_{3}=k_{4}$ $=k_{7}=k_{8}$, a balanced transport. We might have proposed the term symmetrical transport, except that the latter term has already been pre-empted. Stein (1967) defines a symmetrical transport as one having $k_{1}=k_{6}, k_{3}=k_{4}$, $k_{2}=k_{5}$ and $k_{7}=k_{8}$, i.e., a facilitated diffusion for which $K_{\mathrm{m}}$ and $V_{\max }$ are the same in both directions. The three conditions of transport which we will consider, namely trans stimulation, balance and trans inhibition, are thus symmetrical transports under the definition of Stein.

On removing the restriction of balance, conditions of trans stimulation and of trans inhibition are possible. For a system showing a trans stimulation, the rate constants for the translocation of the loaded carrier are assumed to be larger than those of the unloaded carrier, that is $k_{3}=k_{4}>k_{7}=k_{8}$. Conversely, for a system showing a trans inhibition, the rate constants for the movement of the loaded carrier are assumed to be less than those of the empty carrier, $k_{3}=k_{4}<k_{7}=k_{8}$. 
A description of these trans effects has been simulated with an analog computer AD-64. The simulation solves equations (2) through (6) for the condition where the translocation steps are rate-limiting; other conditions are given in Table 1. Expessions for the influx and the efflux of the simulated uptakes were derived using the steady state approximation. The equations

\section{TABLE 1}

\section{Conditions of the analog simulation}

(1) The translocation reactions are rate limiting.

(2) The $K_{\mathrm{m}}$ and $V_{\max }$ of influx are equal to the $K_{\mathrm{m}}$ and $V_{\max }$ of efflux.

(3) $k_{1}, k_{2}, k_{s}$ and $k_{B}$ are equal to each other and to an arbitrary value of 10.

(4) We define the conditions as follows:

(a) for balance, $k_{3}=k_{4}=k_{7}=k_{8}$

(b) for trans stimulation, $k_{3}=k_{4}=5 k_{7}=5 k_{8}$

(c) for trans inhibition, $5 k_{3}=5 k_{4}=k_{7}=k_{8}$.

(5) The simulation is solved when $k_{3}$ and $k_{3}$ are fixed at an arbitrary value of 0.2 and $k_{7}$ and $k_{8}$ varied, or when $k_{7}$ and $k_{8}$ are fixed at a value of 0.2 and $k_{3}$ and $k_{4}$ are varied.

(6) At zero time, no solute is present and all the carrier is in the empty form equally distributed between the two sides of the membrane.

(7) At the start of the program, the solute is introduced at saturating concentration in a medium of infinite volume.

were verified with the computer by individually changing the value of each rate constant and observing the resultant flux. The flux depends on the amount of the loaded carrier available at the respective membrane surfaces and the value of the rate constant for the rate-limiting step. For influx, velocity equals $k_{3}[C S]_{1}$ and for efflux, velocity equals $k_{4}[C S]_{2}$.

\section{Analog Simulation of Net Transport}

The approach toward equilibrium under the three trans conditions can be seen in Figs 2(a) through 4(a). The curves describe the net influx of the solute with time and the rate of the reverse process as the solute accumulates on the inside and begins to exit from the cell. For the curves obtained when $k_{7}$ and $k_{8}$ are fixed and $k_{3}$ and $k_{4}$ are varied, the approach toward equilibrium for the case of trans stimulation is at least twice as fast as for the other cases. The rates of transport, however, depend on the magnitude as well as on the ratio of the rate constants for the translocation process. Indeed by generating the curves when $k_{3}$ and $k_{4}$ are fixed and $k_{7}$ and $k_{8}$ are varied, we come to simulate a transport system showing a trans inhibition whose rates are greater than those of a system showing a trans stimulation. Therefore, to 

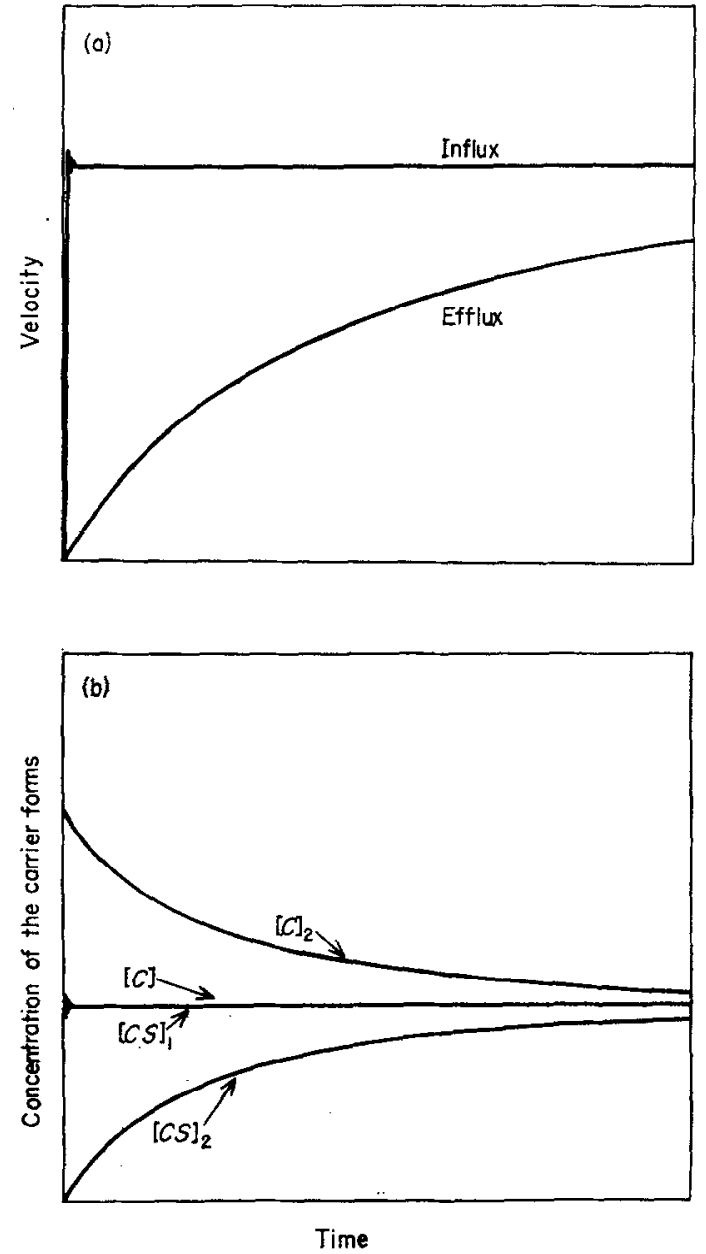

Fig. 2. Computer simulation: progress toward equilibrium under the condition of balance, (a) the fluxes and (b) the distribution of the carrier forms. The rate constants for the translocation of the empty and the loaded carrier are equal.

prevent any bias through the selection of the values for the rate constants, the velocities in Figs 2(a) through 4(a) have been normalized by dividing by the value of $k_{3}$ or $k_{4}$.

Figure 2(a) shows the simulated fluxes under the condition of a balanced transport. After the initial transient period, the inffux remains constant because the condition of balance prevents any displacement of the distribution of the loaded carrier [Fig. 2(b)]. Efflux rises asymptotically as solute accumulates until equilibrium is reached and efflux equals influx. 

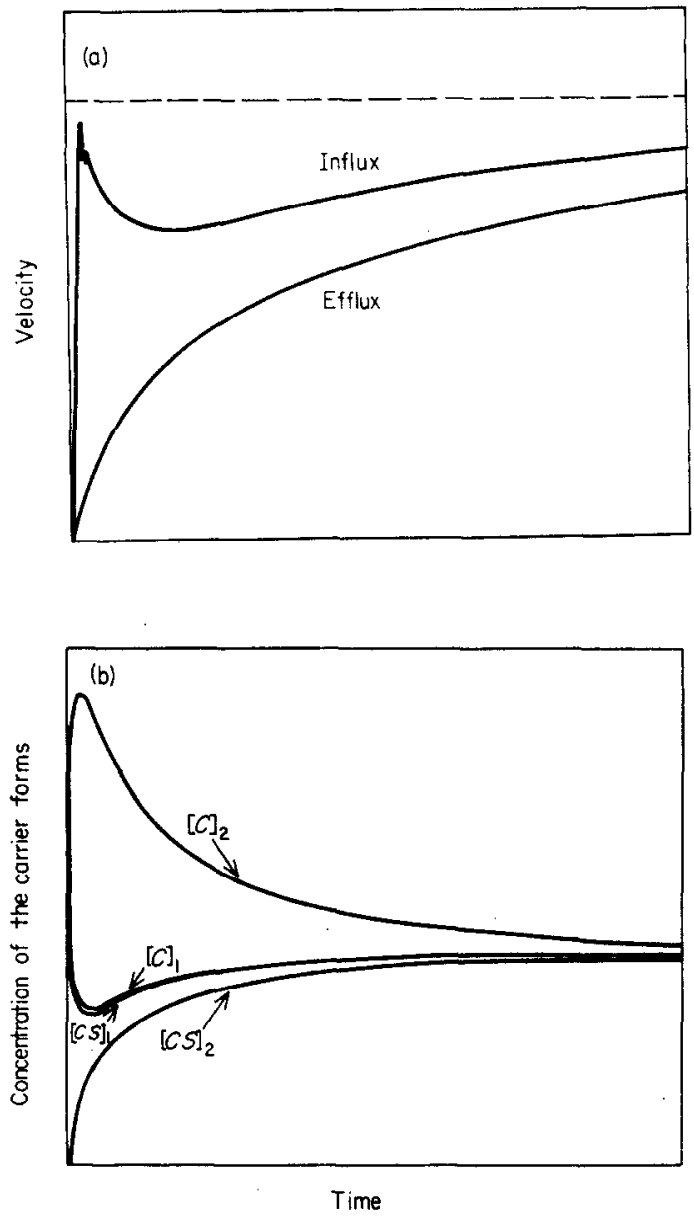

FIG. 3. Computer simulation: progress toward equilibrium under the condition of trans stimulation, (a) the fluxes and (b) the distribution of the carrier forms. The rate constants for the translocation of the loaded carrier are five times the magnitude of those of the empty carrier. The dashed straight line represents the equilibrium state.

The simulation of net uptake under the condition of trans stimulation is seen in Fig. 3. The rapid movement of the loaded carrier across the membrane results in almost an instantaneous maximization of the influx and forces most of the external carrier to the inside. This event depletes the concentration of the external carrier and since the rate of entry is given by the concentration of the loaded carrier, a quick decrease in the influx is also seen.

The decline in the influx is halted as the concentration of the loaded carrier rises on the inner side of the membrane, Since the equilibrium of 

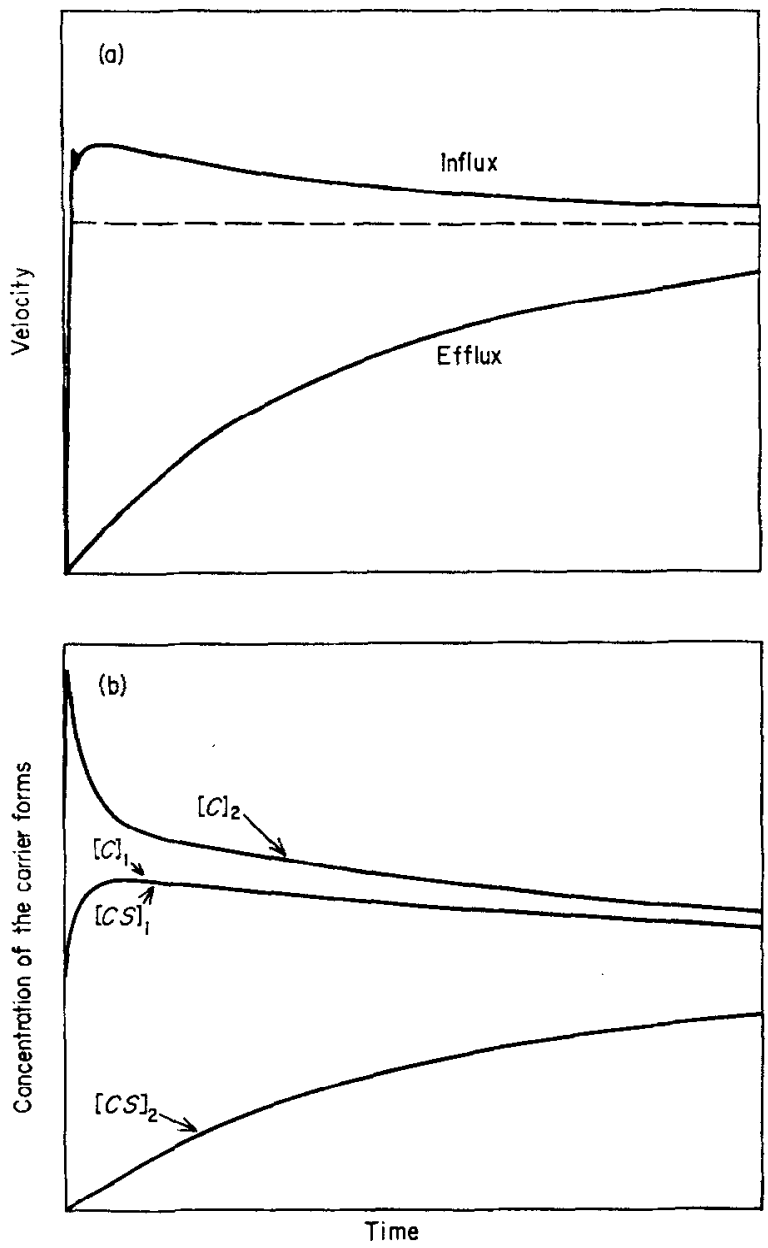

Fig. 4. Computer simulation: progress toward equilibrium under the condition of trans inhibition, (a) the fluxes and (b) the distribution of the carrier forms. The rate constants for the translocation of the loaded carrier are one-fifth the magnitude of those of the empty carrier. The dashed straight line represents the equilibrium state.

carrier species favors the formation of loaded carrier, as solute accumulates the state of the internal carrier rapidly shifts from the empty to the loaded form. The outward movement of the carrier accelerates and helps reduce the asymmetry in the carrier distribution.

Undoubtedly, the pre-steady-state character of the influx is exaggerated in the simulation since the concentration of the carrier was not greatly different from that of the substrate. In a physiological environment, the 
concentration of the carrier may be less than one-millionth of that of the substrate, and the rate constants may accordingly be orders of magnitude greater than that represented on the computer. The approach to a steady state for the binding of solute to the carrier is probably instantaneous in the biological context.

The curves in Fig. 4(a) describe the influx and the efflux under the condition of trans inhibition. The rate-limiting step in the transport here is the slow translocation of the loaded carrier. The carrier combines with the solute and accumulates in the loaded form at the outer surface of the membrane [Fig. 4(b)]. As the uptake continues more of the carrier accumulates in this form, an effect that causes the influx to rise and become higher than the equilibrium influx.

At the inner surface of the membrane, the equilibrium among the forms of the carrier favors the formation of the empty carrier. Most of the loaded carrier will dissociate rather than cross the membrane, for our conditions in the ratio of $250: 1$. Therefore, a large amount of solute must be accumulated before an appreciable internal concentration of the loaded carrier is attained. As the concentration of the loaded carrier at the inner surface gradually increases and the carrier begins to redistribute itself, the rise in the influx is halted and the influx begins to decrease towards the equilibrium value.

The simulated fluxes of trans inhibition and trans stimulation may perhaps best be compared when the translocation of the empty carrier is held constant. In this extreme case, the appearance of solute inside the cell with time is over eight times faster under the condition of trans stimulation than under the condition of trans inhibition. We see how fast solute movement can be under the condition of trans stimulation, how slow uptake can be under the condition of trans inhibition. The rate of movement in a system showing a trans inhibition can be so slow as to give the appearance that the transport is inoperative.

\section{Simulation of Exchange Transport}

The analog computer simulation can be solved for the case where the solute is simultaneously introduced at both sides of the membrane, other conditions in Table 1 remaining the same, so that the phenomenon of exchange transport is simulated. The expected fluxes for an exchange uptake in a system showing a trans stimulation are seen in Fig. 5. Both fluxes are accelerated by the exchange in proportion to the concentration of the load. The initial fall in the influx seen in the absence of internal solute is lessened or eliminated depending on the load, since the exchange will be returning the carrier almost as fast as it enters. 

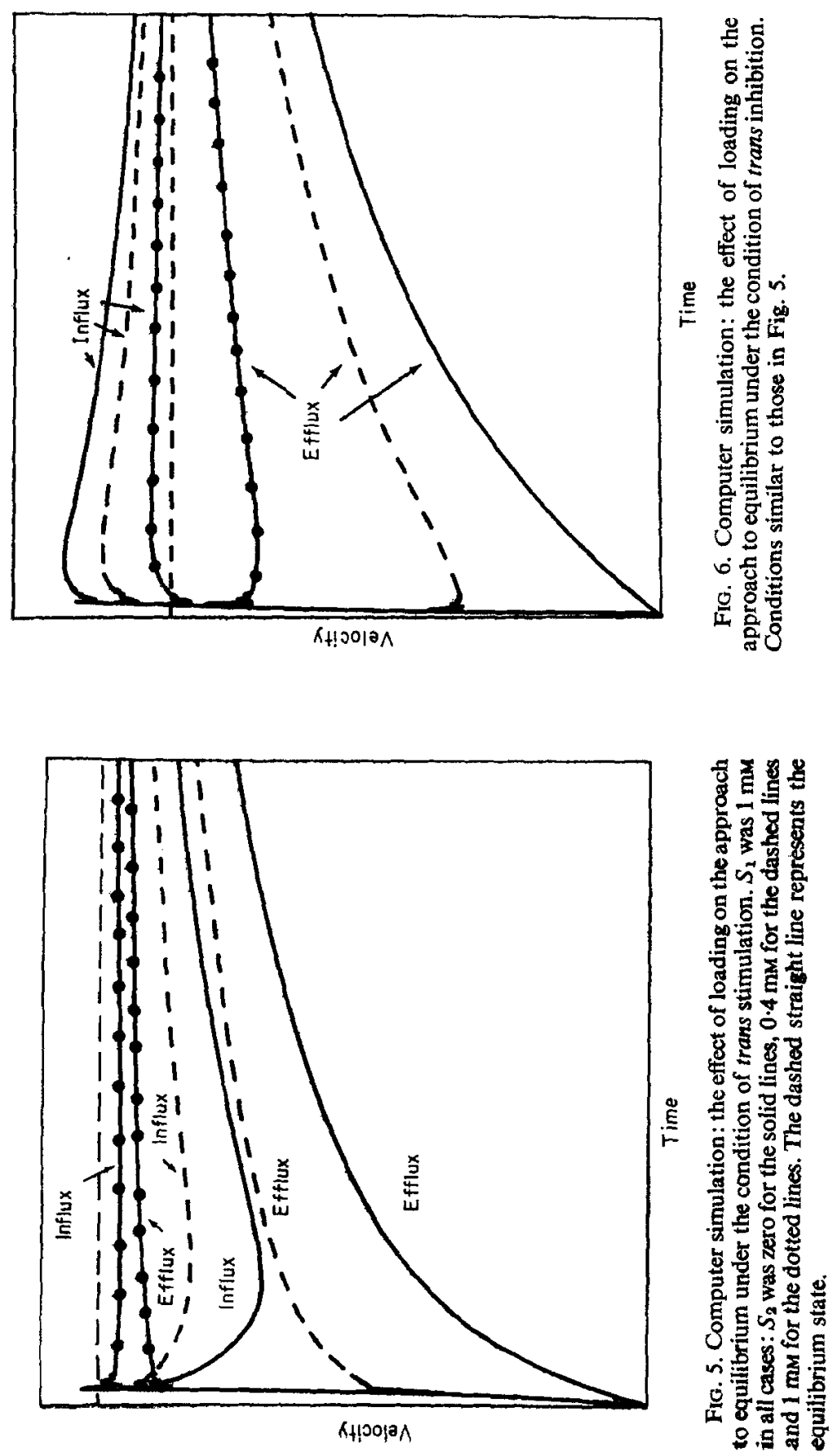
Figure 6 shows the simulation of exchange uptake under the condition of trans inhibition. The exchange increases the efflux but decreases the influx. Because of the intracellular solute already present, carrier asymmetry is not as great as that observed without a load, and the initial rise in the influx above the equilibrium influx is less extreme.

We observe an interesting paradox in the nomenclature of trans inhibition and trans stimulation. Although the influx under the condition of trans inhibition will decrease with the extent of loading of the cell, the computer shows that the pre-equilibrium influx will always be greater than the influx at equilibrium (Fig. 6). Likewise, the influx under the condition of trans stimulation will increase with the extent of loading, yet the pre-equilibrium influx will always be less than the influx at equilibrium (Fig. 5).

\section{Simulation of Tracer Exchange}

To simulate tracer exchange with the analog computer requires that the model in Fig. 1 be modified to describe the transport of two species, $R$ and $S$ (Fig. 7). We assume that $R$ and $S$ are either analogs or different forms of

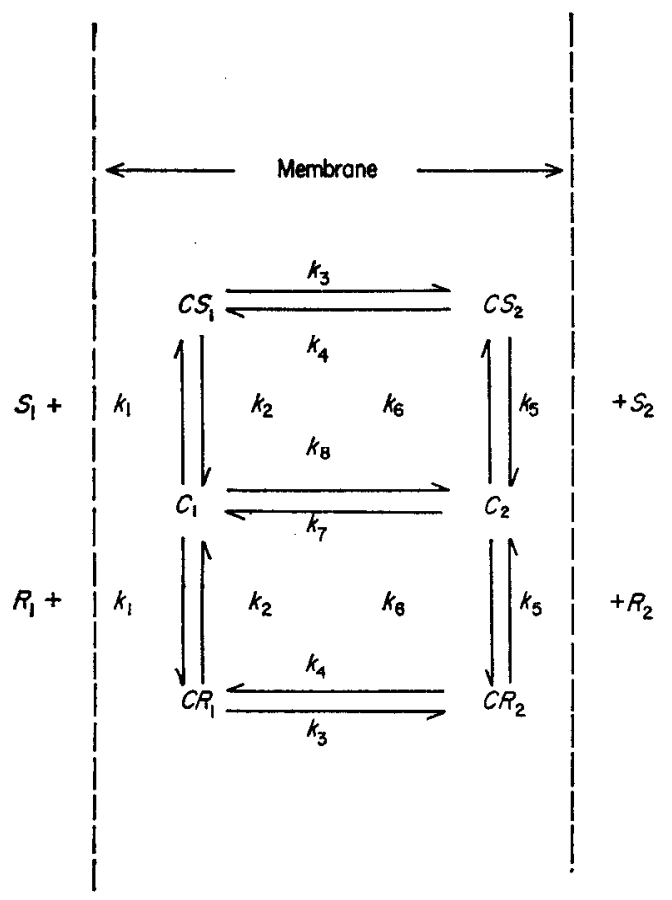

Fig. 7. Proposed model of carrier transport for the competition between two substrates and the carrier. $R$ and $S$ are either analogs or different forms of the same solute. 
the same solute, and in this case specifically, that $S$ is the nonradioactive species and that $R$ is a radioactive tracer.

Since $S_{1} \gg R_{1}, R$ contributes negligibly to the distribution of the carrier forms, and equations (1) through (5) still describe the distribution of $S$ and the carrier forms. At the start of the simulation, the unlabeled solute is simultaneously added to both sides of the membrane but the labeled solute is added only at the outer surface, other conditions in Table 1 remaining the same. The concentrations of $S_{1}$ and $R_{1}$ are again assumed to apply to a medium of infinite volume.

Figure 8 describes the appearance of labeled solute on the inside of the cell with time, with and without the simultaneous addition of a ten-fold higher concentration of solute on the opposite side of the membrane. A large overshoot above the equilibrium concentration of $R$ is seen during exchange

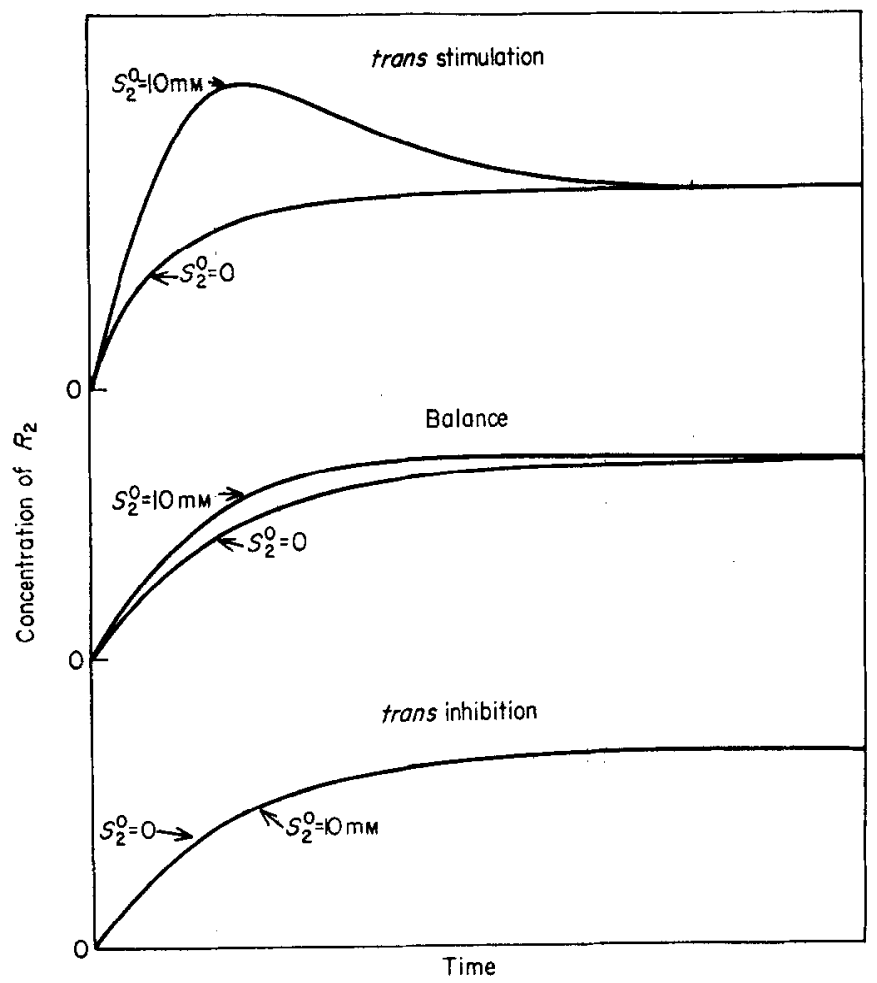

Fir. 8. Computer simulation: appearance of labeled solute inside the cell $\left(R_{2}\right)$ with time under the three conditions. The equations of the model in Fig. 7 were solved when $S_{2}$ was equal to zero (net uptake) or to $10 \mathrm{~mm}$ (exchange uptake); $S_{1}$ was equal to $1 \mathrm{~mm}$ for both cases. 
uptake under the condition of trans stimulation. The shape of the overshoot is consistent with experimental results obtained in this laboratory and elsewhere for trans stimulating systems. A more modest overshoot is seen during exchange uptake under the condition of balanced transport. These overshoots in the concentration of $R$ disappear when the concentration of internal solute is equal to or less than the outside concentration.

The extreme slowness of the net movement of $R$ under the condition of trans inhibition explains why only a slight additional decrease in the transport of $R$ is seen during an exchange uptake. The experimental observation of trans inhibition is diffcult and probably requires both a large concentration of the trans solute relative to the $K_{\mathrm{m}}$, and a large difference in the translocation rate constants for the loaded and the empty carrier. Biological cases of trans inhibition may sometimes be overlooked or confused with a balanced transport through failure to use sufficient levels of substrate in radioactive tracer experiments.

Although we have limited our discussion to passive transports, the results of the analog computer simulation could apply equally well to uphill transport. Asymmetries such as have been suggested by Stein (1967) and by Geck (1971) will not alter the shape of the flux curves, but will change the steady states levels of substrate. Representative solutions for active transport under the condition of trans stimulation are presented in Figs 10 and 11. Similar solutions can be expected for the other conditions.

\section{Trans Stimulation Phenomena in the Ehrlich Cell}

A well characterized laboratory model of trans stimulation is that of system $L$ of the Ehrlich ascites tumor cell (Oxender \& Christensen, 1963). Reactivity with this system is high for the bulky neutral amino acids and for the synthetic amino acid 2-aminonorbornane-2-carboxylic acid (Tager \& Christensen, 1971). It is not safe, however, to assume that system $L$ is a passive system for any given substrate. As has been discussed elsewhere (Christensen, deCespedes, Handlogten \& Ronquist, 1973), substrates with certain structures are strongly concentrated by this system by what may well be an exaggeration of a weak access to energy-coupling for the normal substrates. Nevertheless, trans stimulation by endogenously present amino acids plays a large role in the apparent uphill operation of system $L$.

Transport of the norbornane amino acid shows a vigorous trans stimulation so that net uptake can be demonstrated only with special care (Christensen, deCespedes, Handlogten \& Ronquist, 1974). In order to slow down the exchange process and to help deduce which step in transport was ratelimiting, the Ehrlich cell was modified by treatment with 2, 4-dinitrofluoro- 
benzene (DNFB). Treatment with this chemical at $1 \mathrm{~mm}$ prior to the uptake measurement reduced the initial rate of net and exchange uptake of the norbornane amino acid by approximately $50 \%$, while the steady state level of the amino acid was reduced to only one-sixth the level observed in cells not so treated (Fig. 9).

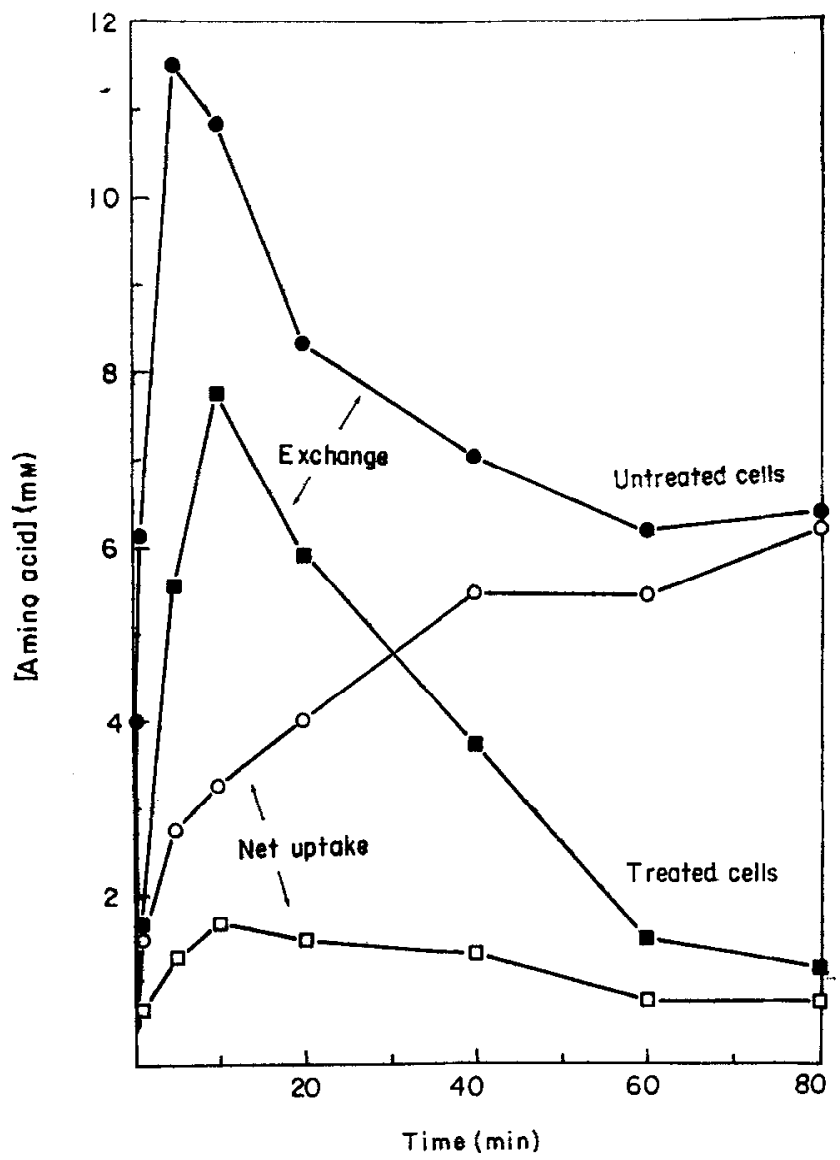

FiG. 9. Uptake of $0.4 \mathrm{~mm}$ norbornane amino acid in Ehrlich ascites tumor cells. For the exchange experiments, the cells were loaded for $20 \mathrm{~min}$ with $10 \mathrm{mM}$ norbornane amino acid. The calculated internal concentration of the norbornane amino acid at the start of the experiment was $15 \mathrm{mM}$. For the net uptake experiments, the exchangeable amino acids in the intra-cellular pool were lowered by repeated incubation in Krebs-Ringer bicarbonate bufier containing $6 \mathrm{~mm} \mathrm{Na}{ }^{+}$and $0.1 \mathrm{mM} \alpha$-aminoisobutyric acid. Each cell population was then divided in half. One half was incubated in 0.3\% alcoholic Krebs-Ringer bicarbonate buffer containing $1 \mathrm{mM}$ DNFB for $2 \mathrm{~min}$; the other half served as the control and was incubated in the same buffer without DNFB. Uptake was measured at $37^{\circ} \mathrm{C}$. 
Assuming provisionally that a change in the steady state level reflects an asymmetric action of DNFB on the transport rate constants, we attempted to simulate this behavior on the computer, using the model of tracer exchange. An asymmetric action of DNFB on the rate constants for the translocation of the empty carrier would result in the uptake behavior seen in Fig. 10. Although the exchange overshoot was greatly reduced and a steady state level only one-fourth that of the control was reached in the treated cells, the simulated effect of DNFB on the initial rates of uptake did not correspond to the experimental behavior. Much better agreement with the experimental data was obtained when we assumed that the chemical acted asymmetrically on either $k_{1}$ and $k_{2}$ or $k_{3}$ and $k_{4}$ (Fig. 11). Additional experiments (not

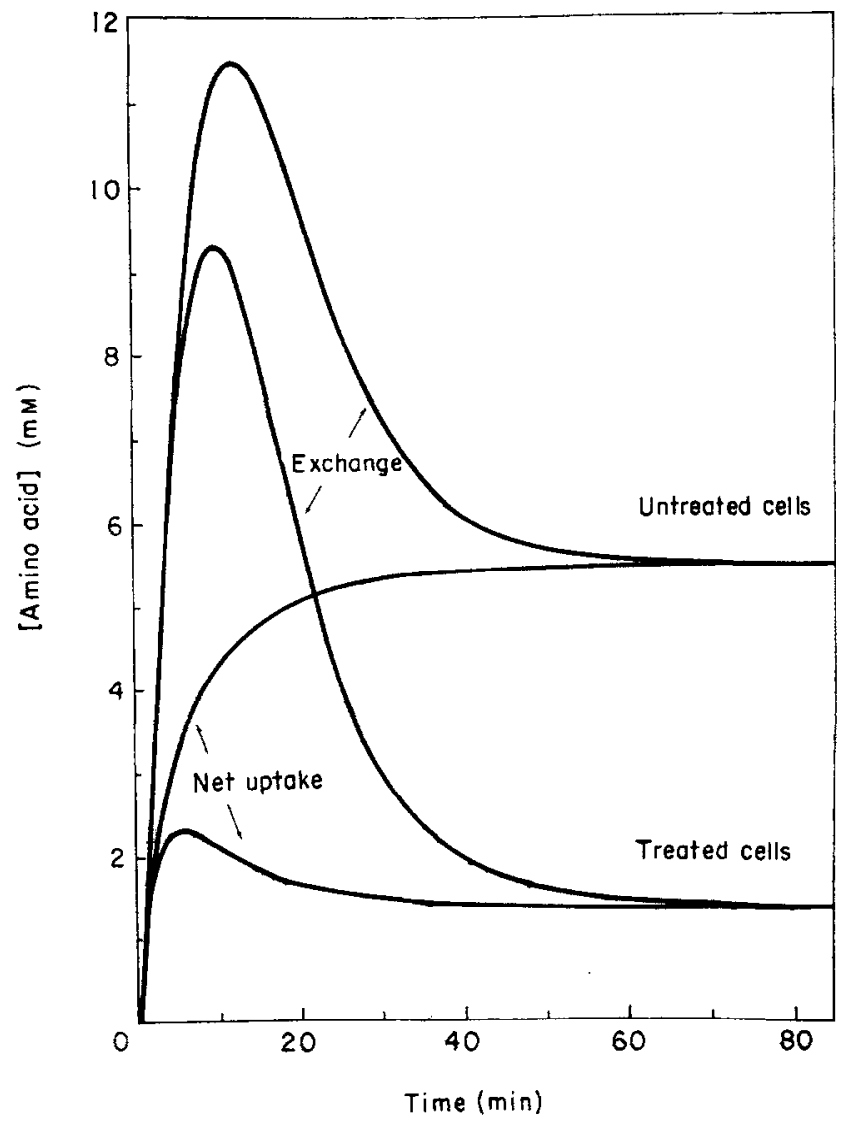

Fig. 10. Computer simulation: appearance of $R_{2}$ with time under the condition of trans stimulation where $k_{7}=k_{8}$ (untreated cells) and where $k_{7}=\frac{1}{2} k_{9}$ (treated cells), Conditions similar to those in Fig. 8 , 


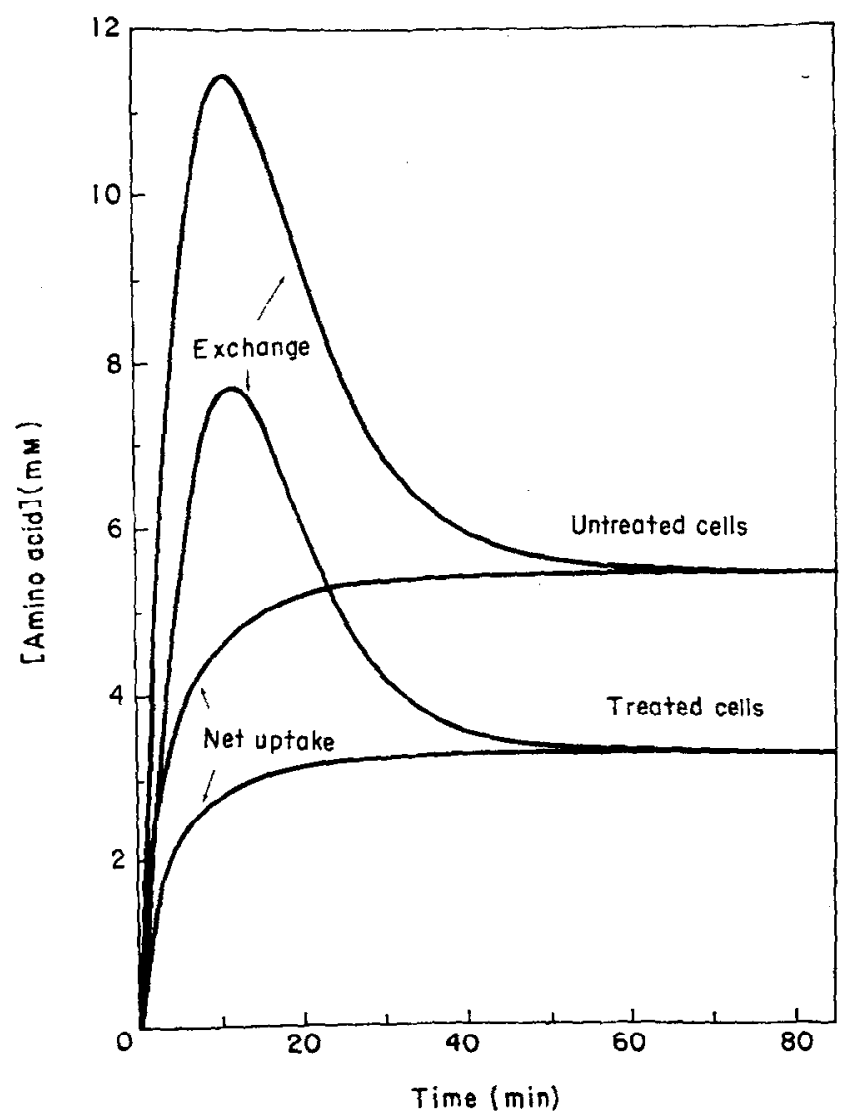

Fig. 11. Computer simulation: appearance of $R_{2}$ with time under the condition of trans stimulation where $k_{3}=k_{1}$ (untreated cells) and where $k_{3}=\frac{1}{2} k_{4}$ (treated cells). The same solution was obtained where $k_{1}=k_{2}$ (untreated cells) and where $k_{1}=1 k_{2}$ (treated cells). Conditions similar to those in Fig. 8.

reported here) showed that $D N F B$ treatment did not affect the $K_{\mathrm{m}}$, but did reduce the $V_{\max }$ of uptake, a result which suggested that the major effect of the chemical was on the movement of the loaded carrier across the membrane.

Whereas the simulation confirms the prediction that a differential slowing of the movements of the loaded and the unloaded carrier can modify the intensity of the trans phenomenon, the sequence of changes produced by DNFB shows that this reagent does not act in that way. Instead, the fit of the simulation model with our experimental results was much better when we assumed that this reagent decreases both $k_{3}$ and $k_{7}$. Figure 12 shows the good fit obtained when $k_{7}$ is decreased twice as much as $k_{3}$. 


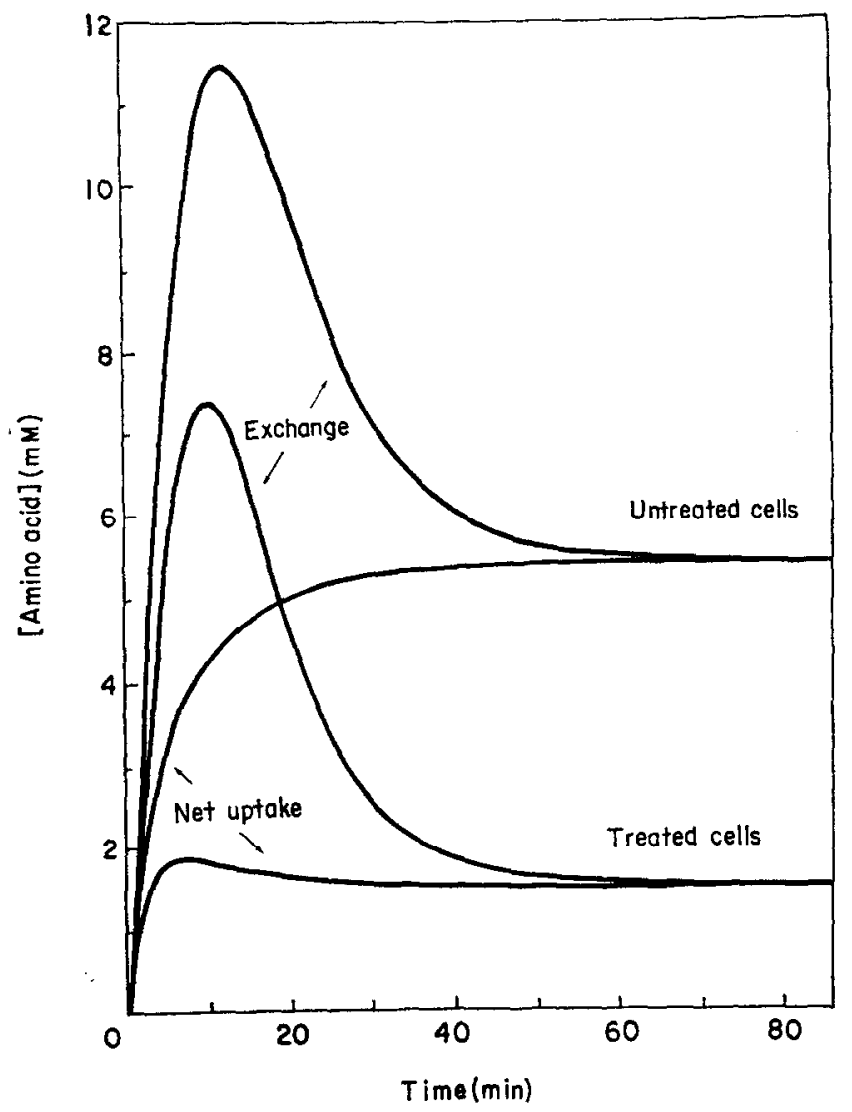

FIo. 12. Computer simulation: appearance of $R_{2}$ with time under the condition of trans stimulation where $k_{3}=k_{4}$ and $k_{7}=k_{\mathrm{a}}$ for the untreated cells and where $k_{3}=0.7 k_{4}$ and $k_{7}=0.4 k_{8}$ for the treated cells. Conditions similar to those in Fig. 8.

The flaw in the earlier interpetation by Oxender \& Whitmore (1966) was that too much attention was given to the rate changes in the first few minutes, too little attention to the lowered steady state toward which the poisoned process was tending (Fig. 9). Effects of DNFB on the steady state are also evident in the Oxender's unpublished results, so that Fig. 12 describes the action of DNFB on the transport of leucine as well as on that of the norbornane amino acid.

For an active transport process, decreasing either $k_{3}$ or $k_{7}$ will diminish the steepness of the uphill transport, effects that might arise through interference with an energy input (Figs 10 and 11). Our hypothesis that both rate constants are decreased by DNFB treatment appears to imply an energy 
input for both of these steps, although we do not take the results of Fig. 12 to establish an unequal energization of the two. Most models of active transport postulate an energy requirement for the translocation of either the loaded or the empty carrier, but not both. Under one extreme type of transport model, the pure vectorial-force model, in which all the external energy serves to drive the empty or the loaded carrier across the membrane,

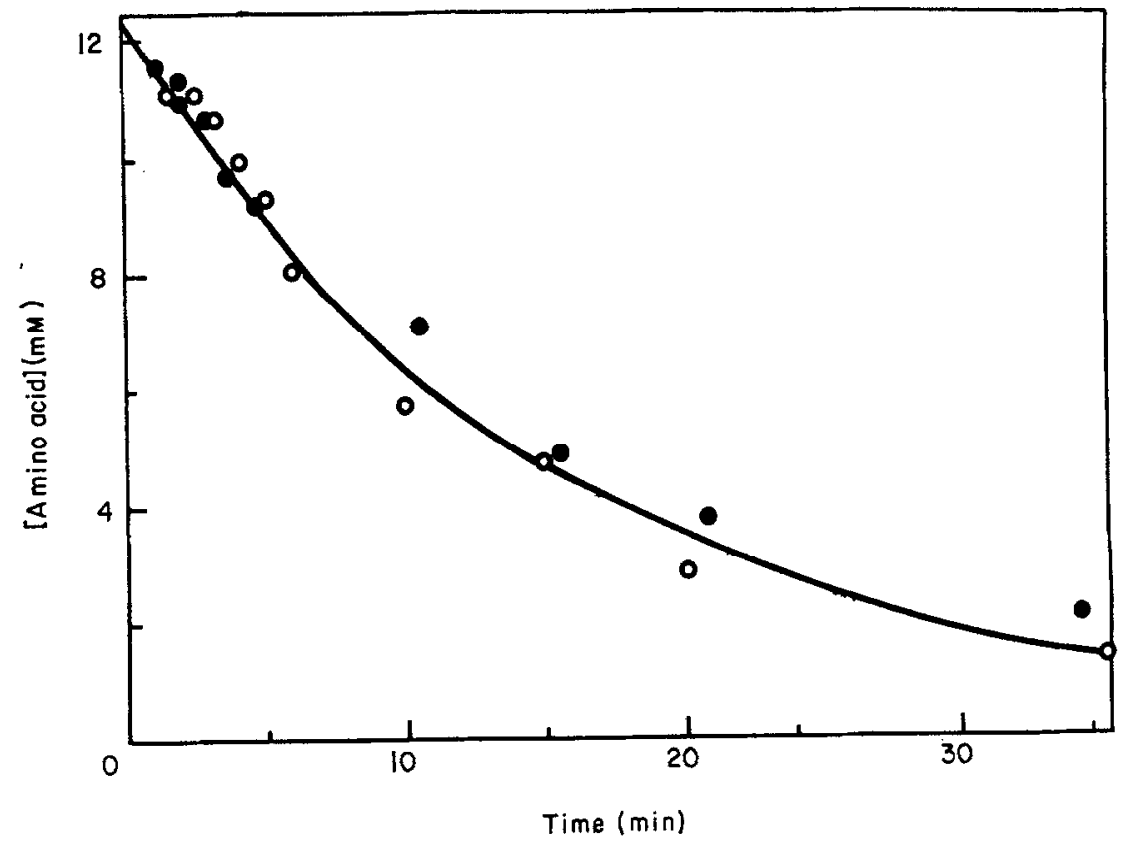

Fig. 13. Time course of the net exodus of the norbornane amino acid at $37^{\circ} \mathrm{C}$ from Ehrlich tumor oelk. Cells were loaded for $20 \mathrm{~min}$ with $10 \mathrm{~mm}$ norboraane amino acid, then treated with or without $1 \mathrm{~mm}$ DNFB as described in Fig 9. The calculated internal concentration of the norbornane amino acid at the start of the experiment was $12.2 \mathrm{~mm}$. Net exodus of the arnino acid was measured into a large volume of medium to minimize recapture. The open circles reprosent the untroated cells; the fillod circles, the cells treated with DNFB.

two sites of energy ooupling might be neoessary to explain our data. Although it would be biologically useful to drive the carrier in the loadod form in one direction and in the unloaded form in the other direction, a mechanistic basis for this duality is not obvious. Under another extrome type of transport model, the pure affinity-change model, in which all the external energy serves to modify the carrier to a form with a different affinity for the substrate (see Christensen, 1975), a single mode of interference with onergy delivery might 
well cause both of the translocation steps productive to uphill transport to appear to be slowed.

Furthermore, under intermediate models in which the external energy serves both to change the affinity of the carrier for the substrate and to provide a rectorial force across the membrane, one might also expect that an interference with energy delivery would decrease the apparent values of both $k_{3}$ and $k_{7}$. In that case these apparent values might show decreases because each may be a product of a true rate constant times an activity coefficient representing the proportion of the carrier in the form contributing to uphill transport. The action of DNFB might then be that of an uncoupling agent with the active form of the chemical serving to modify the asymmetry between the proportions of the carrier presented in its two forms at each surface of the membrane.

As a test of the hypothesis that both $k_{3}$ and $k_{7}$ are affected by DNFB treatment, two predictions can be made concerning exodus: first, if the

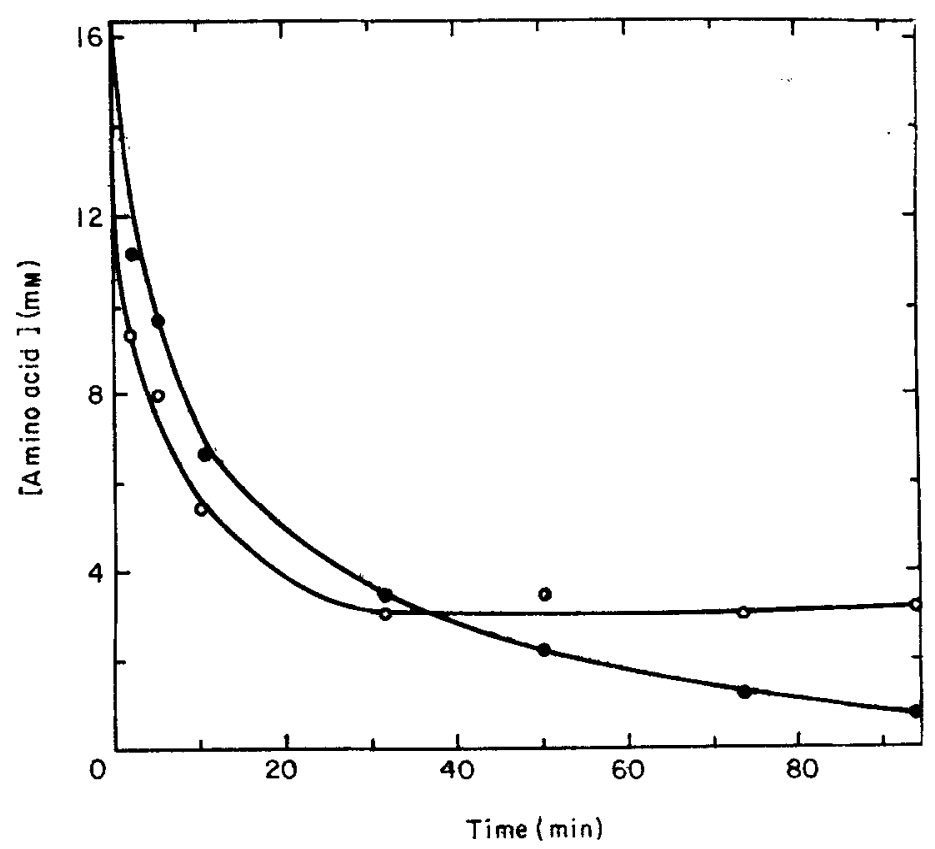

FIG. 14. Time course of the exchanoe exodus of the nombarnme amine acid. The calculated internal concentration of the rarbornane amino acid at the stant of the experiment was $16.3 \mathrm{~mm}$. Exchange exodus was measuned into a small volume of medium containing $0.5 \mathrm{mM}$ norbornane amino acid to maximize recapture of the lont amino acid. Other conditions similar to those in Fig. 13. The open circles represent the urtreated cells; the filled circles, the cells treated with DNFB. 
medium has an infinite volume that net exodus, which is independent of $k_{3}$ and $k_{7}$, should not be affected by the chemical treatment, and second, that exchange exodus, which depends on $k_{3}$ for the exchange stimulation, would be reduced by the treatment. Figures 13 and 14 show that both of these predictions have been confirmed for the exodus of the norbornane amino acid. Note that the rate of recapture of the escaped amino acid, which is directly proportional to $k_{3}$, is also reduced by the treatment (Fig. 14).

It thus seems probable to us that an asymmetric action of DNFB on the movements of the carrier into and out of the Ehrlich cell can account for our results and the preceding observations of Oxender \& Whitmore (1966). Another asymmetric action for this chemical has been reported with sugar transport in the erythrocyte. In this system, which seems to be a balanced transport in our terminology, DNFB appears to bind only to the loaded carrier, and only at the outside surface of the membrane (Krupka, 1971, 1973; Edwards, 1973).

A different means of modifying the relation between the rates of translocation of the empty and the loaded carrier is to modify the structure of the substrate. Christensen (1972) was able to convert the trans stimulation shown by 2 -aminoisobutyric acid into a trans inhibition by $N$-methylating the substrate. Since the transport system was otherwise unmodified in these studies and since a trans inhibition implies that the loaded carrier is moving slower than the empty carrier, we may presume that the effect of $N$-methylating the substrate falls on the magnitude of the rate constants for the translocation of the loaded carrier.

Discussions with and advice from the following members of this University are gratefully acknowledged: Drs D. L. Oxender, G. A. Palmer, and M. A. Savageau.

\section{REFERENCES}

Brrrton, H. G. (1965). J. theor. Biol. 10, 28.

Brock, T. D. \& MoO-PENN, G. (1962). Archs Biochem. Biophys. 98, 183.

Chrustensen, H. N. (1972). Bioenergetics 4, 233.

Christensen, H. N. (1975). Biological Transport, 2nd edn. New York: W. A. Benjamin, Inc. Chrustensen, H. N., deCegpedes, C., Handlocten, M. E. \& Ronquist, G. (1973). Biochim. biophys. Acta 300, 487.

Christensen, H. N., deCrspedes, C., Handlogten, M. E. \& Ronquist, G. (1974). Ann. N.Y. Acad. Sci. 227, 355.

Christrnsen, H. N. \& Handlogten, M. E. (1968). J. biol. Chem. 243, 5428.

EDWARDS, P. A. W. (1973). Biochim. biophys. Acta 307, 415.

GeCK, P. (1971). Biochim. biophys. Acta 241, 462.

Hennz, E. J. \& DuRaIN, R. P. (1957). J, gen. physiol. 41, 101.

HeInz, E. J. \& Walsh, P. M. (1958). J. biol. Chem. 233, 1488.

KoTYK, A. (1973). Biochim. biophys. Acta 300, 183. 
KRUPTA, R. M. (1971). Biochemistry, N.Y. 10, 1143.

KRUPRA, R. M. (1973). Biochim. biophys. Acta 282, 326.

Oxender, D. L. \& Christensen, H. N. (1963). J. biol. Chem. 238, 3686.

Oxender, D. L. \& Whimore, B. (1966). Fedn. Proc. Fedn Am. Socs exp. Biol. 25, 592A. SteIN, W. D. (1967). The Movement of Molecules Across Cell Membranes. New York: Academic Press.

Tager, H. S. \& Christensen, H. N. (1971). J. biol. Chem. 246, 7572.

Thomas, E. L. \& Christensen, H. N. (1971). J. biol. Chem. 246, 1682. 